\title{
Detection of low frequency FGFR3 mutations in the urine of bladder cancer patients using next-generation deep sequencing
}

This article was published in the following Dove Press journal:

Research and Reports in Urology

26 June 2012

Number of times this article has been viewed

\author{
John M Millholland \\ Shuqiang Li \\ Cecilia A Fernandez \\ Anthony P Shuber \\ Predictive Biosciences Inc, \\ Lexington, MA, USA
}

Correspondence: Anthony P Shuber Predictive Biosciences, 128 Spring Street, Lexington, MA 0242I, USA

Tel + I 78| 402 I 780

Fax + I 78| 402 I785

Email tshuber@predictivebiosci.com

\begin{abstract}
Biological fluid-based noninvasive biomarker assays for monitoring and diagnosing disease are clinically powerful. A major technical hurdle for developing these assays is the requirement of high analytical sensitivity so that biomarkers present at very low levels can be consistently detected. In the case of biological fluid-based cancer diagnostic assays, sensitivities similar to those of tissue-based assays are difficult to achieve with DNA markers due to the high abundance of normal DNA background present in the sample. Here we describe a new urine-based assay that uses ultradeep sequencing technology to detect single mutant molecules of fibroblast growth factor receptor 3 (FGFR3) DNA that are indicative of bladder cancer. Detection of FGFR 3 mutations in urine would provide clinicians with a noninvasive means of diagnosing early-stage bladder cancer. The single-molecule assay detects FGFR3 mutant DNA when present at as low as $0.02 \%$ of total urine DNA and results in $91 \%$ concordance with the frequency that FGFR3 mutations are detected in bladder cancer tumors, significantly improving diagnostic performance. To our knowledge, this is the first practical application of next-generation sequencing technology for noninvasive cancer diagnostics.
\end{abstract}

Keywords: FGFR3, mutation, urine, single molecule, sequencing, bladder cancer

\section{Introduction}

Bladder cancer is the seventh leading cause of death in the United States, with approximately 70,000 new cases diagnosed each year. ${ }^{1}$ Although the vast majority of patients with bladder cancer present with low-grade, noninvasive neoplasia (approximately 70\%), recurrence rates for bladder cancer are the highest of any malignancy, and recurrence or progression of the disease is observed in a large percentage of patients. Traditional standard-of-care screening of symptomatic patients, such as those presenting with blood in the urine (hematuria) typically includes cystoscopy, as does surveillance for recurrence of bladder cancer. Noninvasive assays for bladder and other cancers have the potential to improve patient management by reducing discomfort associated with invasive procedures and by the early identification of patients who have a high likelihood of cancer and should receive accelerated intervention.

Activating mutations in FGFR 3 occur in approximately $50 \%$ of all bladder cancers and at higher frequencies in tumors of low-grade and low stage (approximately $60 \%-70 \%))^{2-5}$ There are nine common FGFR3 mutations associated with bladder cancer that are located in three exons, ie, exons 7, 10, and 15, with the exon 7 (S249C) mutation being the most prevalent (about 62\%)., 2,4,67 Therefore, a urine-based noninvasive assay which included FGFR3 mutation detection would significantly increase the detection of early-stage bladder cancer. 
Currently, the most clinically sensitive assays for bladder cancer are based on direct molecular analysis of tumor tissue. Due to the high representativeness of tumor cells present within tissue samples, it is not necessary for these tissue-based assays to have high analytical sensitivity, whereas urine DNA is made up predominantly of normal urothelial DNA, thus requiring a significant increase in analytical sensitivity in order to detect the presence of mutations associated with bladder cancer. Our group and others have developed polymerase chain reaction (PCR)-based assays to detect FGFR3 mutations in the urine of bladder cancer patients. ${ }^{8,9}$ However, these urine-based assays have been limited by the technical ability to detect rare events in a dilute medium where there is a high background of normal DNA. In these assays, FGFR3 mutations are generally found in about $30 \%$ of the urine samples, which is $<50 \%$ concordance with the expected detection in tissue. ${ }^{8,9}$ Others have demonstrated that multiple redundant sampling from a single patient may increase the probability of a positive result. ${ }^{9}$ Although this approach can successfully increase sensitivity, it is not applicable to a commercial setting.

Our goal was to develop an assay that could detect very low levels of FGFR3 mutant DNA in a single urine sample. Here, we describe an ultradeep amplicon sequencing technique that increases FGFR3 mutation detection in urine to $>50 \%$, close to the expected detection rate if every mutation found in tissue could be detected in urine.

\section{Materials and methods Samples}

Urine samples were collected from various academic institutions and urology practices. All participating sites received approval from the appropriate institutional review board and subjects gave their informed consent. All patients in this study had bladder cancer or were undergoing routine evaluation for bladder cancer based on a finding of hematuria (blood in the urine). Of these, bladder cancer was confirmed by pathology in 43 patients, while 24 were found to be negative for cancer by cystoscopy (hematuria+/cystoscopy-). No additional clinical information was used in this study. Urine samples were stabilized with $25 \mathrm{mM}$ ethylenediamine tetra-acetic acid, aliquoted, and stored at $-80^{\circ} \mathrm{C}$. Matching formalin-fixed, paraffinembedded (FFPE) tumor tissue was available for 19 of the patients in this study. An additional 149 FFPE bladder cancer tissue samples were purchased from external sources (Folio Biosciences, Powell, OH; Indivumed, Hamburg, Germany; Erasmus MC, Rotterdam, The Netherlands), and were used to determine the clinical performance of the FGFR 3 quantitative PCR assay in tissue.

\section{DNA isolation from urine}

DNA isolation was performed using the QIAamp MinElute virus vacuum kit (Qiagen, Valencia, CA) with the following modifications: Qiagen protease $(200 \mu \mathrm{L})$ was added to each sample (4 mL of urine), followed by AL lysis buffer ( $4 \mathrm{~mL})$. Following incubation at $56^{\circ} \mathrm{C}$ for 20 minutes, $5 \mathrm{~mL}$ of $100 \%$ molecular biology grade ethanol (Sigma, St Louis, MO) was added, and samples were incubated at room temperature for 5 minutes. Samples were serially loaded onto columns in a vacuum manifold and the columns were washed with $600 \mu \mathrm{L}$ of AW1, AW2, and 100\% ethanol. DNA was eluted in $150 \mu \mathrm{L}$ of water and stored at $-20^{\circ} \mathrm{C}$ until analysis. All samples contained a minimum of $50 \mathrm{ng}$ of purified DNA. For samples analyzed by sequencing, all eluted DNA was lyophilized using a Savant AES1010 vacuum concentrator (Thermo Fisher Scientific, Waltham, MA), and resuspended in $20 \mu \mathrm{L}$ water prior to PCR amplification.

\section{DNA isolation from tissue}

Three $5 \mu \mathrm{m}$ sections of FFPE bladder cancer tumor tissue were pooled and DNA was isolated using a QIAamp DNA FFPE tissue kit (Qiagen) with the following modifications. During deparaffinization, the centrifugation step was increased to 10 minutes after treatment with xylene and subsequent $100 \%$ ethanol washes. In addition, samples were incubated with proteinase $\mathrm{K}$ for 16 hours prior to further processing to ensure complete digestion of tissue. DNA samples were then processed according to the manufacturer's recommendations, and the DNA was eluted in $80 \mu \mathrm{L}$ of ATE buffer (Qiagen).

\section{Primary FGFR3 PCR assay}

Multiplex primary PCR of genomic DNA samples was carried out using chimeric oligonucleotide primers containing 18-22 nucleotides specific for human FGFR3 (exons 7, 10, and 15 ), as well as a 22-nucleotide universal priming sequence that simplifies the multiplexing process. Fluorescent oligonucleotide probes specific for each exon were also included to permit quantitation of each exon. Oligonucleotide primers and fluorescently labeled probes were designed using the Oligo 7.0 program (MBI Inc, Cascade, CO) and synthesized by Integrated DNA Technologies Inc (Coralville, IA).

Primary PCR amplification was performed using a LightCycler 480 II (Roche, Indianapolis, IN) using standard conditions $\left(95^{\circ} \mathrm{C}\right.$ for 10 minutes, followed by 50 cycles of $95^{\circ} \mathrm{C}$ for 10 seconds, $65^{\circ} \mathrm{C}$ for 30 seconds, and $72^{\circ} \mathrm{C}$ for 10 seconds). FastStart Taq polymerase, dNTP mix, and reaction buffers were from Roche Applied Science. Primer, probe, 
and enzyme concentrations were optimized independently for DNA derived from urine and FFPE tissue sections. $20 \mu \mathrm{L}$ of each DNA sample was used as a template for the reaction. After the initial PCR, samples were treated with ExoSAP-IT (Affymetrix/USB Santa Clara, CA) according to the manufacturer's protocol, to remove unincorporated nucleotides and primers and dual-labeled probes prior to using the mutation detection method described below.

\section{Real-time quantitative PCR with locked nucleic acid-blocking oligonucleotides}

FGFR3 mutations were detected utilizing PCR-clamping methodology. Wild-type blocking oligonucleotides containing locked nucleic acid bases surrounding known mutation sites were included along with real-time PCR primers and dual-labeled fluorescent probes. All oligonucleotide primers and fluorescent probes were synthesized by Integrated DNA Technologies Inc. Locked nucleic acid-containing oligonucleotides were obtained from Exiqon, Inc (Woburn, MA). Duplex real-time PCR reactions were designed to detect eight known FGFR3 mutations. Reaction 1 contained primers, probes, and locked nucleic acid-blocking oligonucleotides to detect two exon 7 mutations (R248C and S249C), and one exon 10 mutation (Y375C). Reaction 2 was similarly designed to detect one exon 10 mutation (G372C), and four exon 15 mutations (K652M, K652T, K652E, and K652Q).

Prior to mutation detection PCR, templates for reactions 1 and 2 were diluted $1: 10,000$ and 1:5000 in $\mathrm{H}_{2} \mathrm{O}$, respectively. All real-time mutation detection PCRs were carried out using the LightCycler 480II instrument. FastStart Taq polymerase, dNTP mix, and reaction buffers were from Roche Applied Science. Duplex real-time PCR reactions, with and without locked nucleic acid-blocking oligonucleotides, were assembled in duplicate for each amplification. Each reaction also contained $5 \mu \mathrm{L}$ of diluted primary PCR product in a final volume of $50 \mu \mathrm{L}$. PCR reactions with locked nucleic acidblocking oligonucleotides contained a final concentration of $4 \mu \mathrm{M}$ (exon 7), $4 \mu \mathrm{M}$ (exon 10, G372C), $7 \mu \mathrm{M}$ (exon 10, Y375C), and $20 \mu \mathrm{M}$ (exon 15). All samples for each duplex mutation detection reaction (with and without locked nucleic acid-blocking oligonucleotides) were amplified in a single 96-well PCR plate, with the same PCR conditions of $95^{\circ} \mathrm{C}$ for 10 minutes, followed by 50 cycles of $95^{\circ} \mathrm{C}$ for 10 seconds, and $60^{\circ} \mathrm{C}$ for 30 seconds, and $72^{\circ} \mathrm{C}$ for 10 seconds.

Positive control plasmids were designed to incorporate the region of the mutation, as well as flanking sequences to facilitate amplification of the positive control plasmid during the primary PCR step described above. Each positive control plasmid contained a single nucleotide change, confirmed by sequencing. All plasmids were synthesized by GENEART AG (Regensburg, Germany). Copy number was determined using standard methods, and plasmids were diluted with human genomic DNA accordingly to achieve the desired final concentration of $1 \%$. Each PCR plate contained a negative mutation control (human genomic DNA) and a representative positive mutation control (1\% mutant DNA in a background of normal human genomic DNA) for each exon tested. Data analysis was carried out using the advanced relative quantitation module in the LightCycler software package (v1.5), using the negative mutation controls amplified in the presence and absence of locked nucleic acid-blocking oligonucleotides as target/reference calibrators, respectively. A ratio between these two amplification curves was determined, giving a normalized value of 1.0. All other samples in a given assay were compared with this calibrator control, and assigned a normalized ratio. Any samples with a normalized ratio above established cutoffs were reviewed to determine if the sample was positive for a mutation.

\section{Ultradeep amplicon sequencing: initial PCR amplification}

Control human genomic DNA (Promega, Madison, WI) or DNA isolated from urine (as described above) was amplified using chimeric primers containing sequences specific for $F G F R 3$ exons 7, 10, and 15 . The sequence-specific portion of these primers was identical to that used in the FGFR3 quantitative PCR assay described above. In addition, these primers contain specific adapters for unidirectional sequencing on the Roche GS Junior platform, including a four base pair library key, and a 10 base pair barcode sequence used to permit analysis of multiple samples per sequencing run. The PCR reaction was performed using the LightCycler 480 with the conditions outlined for the primary PCR amplification in the FGFR 3 quantitative PCR assay as detailed above.

After the initial PCR amplification, amplicons were purified with AMPure XP magnetic beads (Beckman Coulter, Brea, CA) according to the manufacturer's instructions. The purified amplicons were quantitated using a Nanodrop 2000 ultraviolet spectrophotometer (Thermo Fisher Scientific), and the copy number was calculated based on the average length of the PCR products in each multiplex amplification using standard techniques. Each sample was diluted to $1 \times 10^{9}$ copies $/ \mu \mathrm{L}$, pooled with the appropriate number of additional samples, and diluted to a final working concentration of $1 \times 10^{6}$ per $\mu \mathrm{L}$. This stock was then used as a template for an emulsion PCR using the GS Junior Titanium emPCR 
Lib-L kit (Roche 454), which was carried out according to the manufacturer's directions for sequencing amplicon libraries, with three modifications. First, the ratio of DNA to beads was modified to $1: 1$, the amount of AMP primer was decreased to $10 \mu \mathrm{L}$, and the template was incubated at $95^{\circ} \mathrm{C}$ for 2 minutes and then kept at $4^{\circ} \mathrm{C}$ for 10 minutes prior to DNA binding to capture beads. After emulsion PCR, the DNA-containing beads were enriched, and prepared for sequencing. All steps in the sequencing portion of the assay were performed as suggested by the manufacturer, using the GS Junior Titanium Sequencing Kit (Roche 454). The presence of mutations in exon 7, 10, and 15 was determined using the AVA software package (Roche 454).

Several thresholds were applied to the data to determine if a sample contained a mutation in FGFR3 exons 7, 10, or 15 . We determined the frequency of false-positives (background noise) for each mutation site by repeatedly sequencing control wild-type human genomic DNA. For an experimental sample to be considered positive, a minimum of three sequencing reads that identified a mutation of interest was required, and the frequency of the mutant reads had to be higher than that of the background.

\section{Results}

\section{FGFR3 mutation detection by quantitative PCR}

The real-time quantitative PCR assay uses locked nucleic acid oligonucleotides to amplify normal and mutant FGFR3 DNA differentially from tumors. The quantitative PCR assay was designed and optimized to detect mutant FGFR3 DNA when it was $\geq 1 \%$ of the DNA sample. This assay detected FGFR3 mutant DNA in tumor samples with a sensitivity of $61.7 \%$ (Table 1), comparable with that found in other studies. ${ }^{5,10-13}$ However, in urine, the sensitivity of this assay was $11.6 \%$ (Table 1) which is not concordant with the informativeness of FGFR3 mutations found in tissue, indicating that we

Table I Sensitivity of quantitative polymerase chain reaction assay in tumor tissue and urine samples from patients with different stages of bladder cancer

\begin{tabular}{lll}
\hline Stage & Sensitivity & \\
\cline { 2 - 3 } & Tumor tissue & Urine \\
\hline $\mathrm{Ta}$ & $66.7 \%(82 / / 23)$ & $\mathrm{II} .1 \%(3 / 27)$ \\
$\mathrm{TI}$ & $38.5 \%(10 / 26)$ & $22.2 \%(2 / 9)$ \\
$\geq \mathrm{T} 2$ & $\mathrm{NA}$ & $0 \%(0 / 7)$ \\
All stages & $61.7 \%(92 / 149)$ & $11.6 \%(5 / 43)$ \\
& $(95 \% \mathrm{Cl} 53 \%-70 \%)$ & $(95 \% \mathrm{Cl} 5 \%-24 \%)$ \\
\hline
\end{tabular}

needed to use a different technical approach with higher analytical sensitivity.

\section{Single molecule assay for detecting low levels of mutant DNA}

We evaluated whether ultradeep amplicon sequencing technology would have greater sensitivity for detecting mutant FGFR3 DNA in urine. Specifically, we utilized a Roche 454 GS Junior sequencer, which uses a large-scale parallel pyrosequencing system capable of sequencing roughly 25 megabases of DNA. FGFR3 exon-specific amplicons containing sequencing adapters at the $5^{\prime}$ and $3^{\prime}$ ends are generated by multiplex real-time PCR. These amplicons are then fixed, in a specific ratio, to DNA-capture beads in a water-in-oil emulsion PCR. After purifying the DNA-containing capture beads, sequencing is carried out in a maximum of approximately 250,000 individual wells of a picotiter plate.

Although the analytical sensitivity of this system is reported to detect specific DNA molecules as low as $1 \%$, in theory, single molecule analysis should permit detection of molecules present at much lower frequencies. Hence, we optimized the existing protocols to improve the analytical sensitivity by about 500 -fold (about $0.02 \%$ ).

The analytical sensitivity of this technology is dependent upon the number of overall sequencing reads per urine sample; the higher the number of reads the greater the likelihood of detecting a rare molecule. To increase the number of reads, we varied the ratio of DNA molecules per bead and also the reaction conditions (Table 2). We found that a bead ratio of $1: 1$ and inclusion of a $95^{\circ} \mathrm{C}$ heating step prior to fixing the DNA to the beads resulted in the highest number sequencing reads $(175,991)$ and the lowest percentage

Table 2 Assay optimization

\begin{tabular}{|c|c|c|}
\hline $\begin{array}{l}\text { Optimizing } \\
\text { condition }\end{array}$ & $\begin{array}{l}\text { Sequence reads } \\
\text { per experiment }\end{array}$ & $\%$ dot + mixed $^{a}$ \\
\hline \multicolumn{3}{|c|}{ Ratio of DNA molecule/bead } \\
\hline 2:1 & 78,917 & 11.49 \\
\hline I.5:1 & 74,254 & 9.89 \\
\hline $1: 1$ & 83,757 & 6.70 \\
\hline $0.5: 1$ & 72,245 & 6.13 \\
\hline \multicolumn{3}{|c|}{ Heating prior to bead annealing } \\
\hline No heating & 83,757 & 6.70 \\
\hline $95^{\circ} \mathrm{C}, 2$ minutes & $|75,99|$ & 1.48 \\
\hline
\end{tabular}

Notes: ${ }^{a} \%$ Dot + mixed is an aggregate percentage of two measurements and is indicative of the percentage of nonanalyzable wells. \% Dot represents sequencing reads with at least successive reads in which a base was not incorporated (possibly due to short templates or failed initial amplification) and "mixed" represents wells with too many nucleotides incorporated due to having more than one template/bead, noise from neighboring wells that interfere with analysis, or a low signal-to-noise ratio; 'ratio of beads to molecules of DNA was I:I.

Abbreviations: $\mathrm{Cl}$, confidence interval; $\mathrm{NA}$, not assayed. 
of noninterpretable reads (1.48\%). Because this optimized ultradeep sequencing method evaluates the sequence of single molecules of FGFR3, we refer to the assay as single-molecule FGFR3. This assay can detect the nine most common FGFR3 mutations.

To determine the analytical sensitivity of single-molecule FGFR3 for mutations in each exon, a plasmid containing a known mutation in exons 7,10 , or 15 was titrated into normal human genomic DNA. We found that mutations in exons 7 , 10 , and 15 were detected above background when the mutant sequence was $0.02 \%, 0.02 \%$, and $0.01 \%$, respectively, of the total DNA (Table 3). This level of sensitivity is greater than what has been previously reported in cancer assays based on bodily fluids. ${ }^{9,14,15}$

Because the nine mutations of interest are found in three separate exons, we next tested whether analysis of all three exons could be multiplexed without losing analytical sensitivity. Plasmid DNA containing the exon 7 S249C mutation was added to control genomic DNA to a final concentration of about $0.02 \%$ and the amplification was multiplexed using primers for all three exons. Similar to the nonmultiplexed reaction, the multiplexed assay detected exon 7 S249C mutant DNA at $0.02 \%$ of the total DNA.

\section{Mutation detection using single-molecule FGFR3 concordant with tissue mutations}

Given the increased sensitivity of this assay, the analytical background for each mutant site needed to be established within sequences known not to have a mutation. Therefore, we sequenced control human genomic DNA in multiple experiments and established thresholds for the background frequency of each mutation (Supplemental Table 1). A urine sample was considered positive for an FGFR3 mutation if the

Table 3 Determining assay sensitivity

\begin{tabular}{llll}
\hline & $\begin{array}{l}\text { Exon-specific } \\
\text { reads }\end{array}$ & $\begin{array}{l}\text { Mutant-positive } \\
\text { reads }\end{array}$ & $\begin{array}{l}\text { \% mutant } \\
\text { detected }\end{array}$ \\
\hline \multicolumn{2}{l}{ For each } & individual exon \\
Exon 7 & 38967 & & $0.02 \%$ \\
Exon 10 & 56657 & 10 & $0.02 \%$ \\
Exon 15 & 26972 & 2 & $0.01 \%$ \\
Multiplex assay with exon $7^{\mathrm{b}}$ & & $0.02 \%$ \\
Exon 7 & 34489 & 6 & 0 \\
Exon 10 & 24202 & 0 & 0 \\
Exon 15 & 9975 & 0 &
\end{tabular}

Notes: aPlasmid DNA containing FGFR3 sequence with a single mutation from one exon was added to human genomic DNA such that it was about $0.02 \%$ of the samples. The assay contained only primers for the particular exon being tested; bplasmid DNA containing FGFR3 sequence for mutant exon 7 was added to human genomic DNA such that they represented approximately $0.02 \%$ of the sample. The assay was performed with primers for all three exons. frequency of the mutant reads was above the normal DNA thresholds and if there were at least three mutant sequencing reads per sequencing experiment.

To determine whether $0.02 \%$ sensitivity of the singlemolecule assay in urine samples was sufficient to detect most FGFR 3 mutant tumors, we evaluated the presence of FGFR3 mutations in matched tumor and urine samples from 19 bladder cancer patients. Using the quantitative PCR assay to determine the mutation status of the tissue samples, 11 of the 19 tumors were determined to have an FGFR 3 mutation (Table 4). However, in urine, only six of 19 samples were positive for a mutation by quantitative PCR, while 15 of the 19 urine samples were positive by small-molecule FGFR3 (Table 4). Importantly, of the 11 tumor samples identified as FGFR3 mutant-positive, 10 were also positive in urine by small-molecule $F G F R 3$, indicating a $91 \%$ concordance between tissue and urine (Table 4). In contrast, only five of these tissue-positive samples were positive by the FGFR3 quantitative PCR assay on the corresponding urine sample (46\% concordance).

\section{Small-molecule FGFR3 improves clinical performance in urine}

To determine the sensitivity of this new small-molecule FGFR 3 assay in a clinical setting, we evaluated urine samples from bladder cancer patients $(\mathrm{n}=43)$ by both smallmolecule $F G F R 3$ and quantitative PCR. Each urine DNA sample was assayed for FGFR3 mutations in exons 7, 10, or 15. The samples were representative of various stages of bladder cancer, with most having early nonmuscle invasive disease (Ta and T1). Results of a representative smallmolecule FGFR3 experiment are shown in Supplemental Table 2. Of the samples analyzed, the quantitative PCR assay identified five as positive for FGFR 3 mutations. The small-molecule $F G F R 3$ assay identified these five patients and also an additional 19 patients as having FGFR3 mutant DNA (Table 5) that were present at $<1 \%$ and as low as $0.02 \%$ of the total urine DNA. The clinical sensitivity of small-molecule $F G F R 3$ was $55.8 \%$, more representative of the frequency of FGFR 3 mutations detected in tumor tissues. In contrast, the quantitative PCR assay had lower analytical sensitivity and resulted in a clinical sensitivity of only $11.6 \%$.

To determine whether increased analytical sensitivity of the small-molecule FGFR3 assay would affect clinical specificity, we analyzed the urine of 24 individuals who had hematuria but who did not have bladder cancer (as determined by cystoscopy). None of the 24 patients were found to have 
Table 4 Summary of the sensitivity and concordance of analysis of DNA isolated from urine by quantitative polymerase chain reaction and small-molecule FGFR3

\begin{tabular}{llll}
\hline & qPCR tumor & qPCR urine & smFGFR3-urine \\
\hline Sensitivity & $58 \%(1 \mathrm{I} / 19)$ & $32 \%(6 / 19)$ & $79 \%(I 5 / 19)$ \\
& $(95 \% \mathrm{Cl} \mathrm{36 \% -77 \% )}$ & $(95 \% \mathrm{Cl} 15 \%-54 \%)$ & $(95 \% \mathrm{Cl} 57 \%-92 \%)$ \\
Concordance & - & $46 \%(5 / 11)$ & $91 \%(10 / 11)$ \\
& & $(95 \% \mathrm{Cl} 21 \%-72 \%)$ & $(95 \% \mathrm{Cl} 62 \%-98 \%)$ \\
\hline
\end{tabular}

Abbreviations: $\mathrm{Cl}$, confidence interval; qPCR, quantitative polymerase chain reaction; smFGFR3, small-molecule FGFR3.

FGFR3 mutations by the small-molecule FGFR3 assay, resulting in $100 \%$ specificity.

\section{Discussion}

We used ultradeep sequencing technology to develop a urinebased assay, capable of being multiplexed, for detecting early bladder cancer that has high clinical sensitivity (about 60\%) and is $91 \%$ concordant with tumor tissue results. Interestingly, the single-molecule assay identified five additional urine DNA samples not identified as carrying FGFR3 mutations by quantitative PCR in tissue, possibly reflecting sampling issues related to tumor heterogeneity ${ }^{16}$ or stochastic sampling and suggesting that with such high analytical sensitivity, a noninvasive urine assay may be more representative of the entire urothelium than analysis of tumor or biopsy sections.

Clinical performance is strongly influenced by differences among patients in biomarker levels that may depend upon tumor stage, type, and mutational status. Although the smallmolecule FGFR 3 assay has different analytical thresholds for each mutation, we are able to detect each mutation well below a $1 \%$ mutant fraction, and this analytical sensitivity is sufficient to deliver clinical performance in urine that is comparable with that in tissue. While our quantitative PCR assay has sufficient analytical sensitivity to detect FGFR3 mutations in tissue where the mutant to normal ratio is high,

Table 5 Summary of small-molecule FGFR3 assay sensitivity by stage and grade in urine samples from 43 bladder cancer patients

\begin{tabular}{lll}
\hline & qPCR & smFGFR3 \\
\hline Tumor stage & & \\
Ta & $11.1 \%(3 / 27)$ & $63.0 \%(17 / 27)$ \\
TI & $22.2 \%(2 / 9)$ & $55.6 \%(5 / 9)$ \\
Tis, T2, T3 & $0 \%(0 / 7)$ & $28.6 \%(2 / 7)$ \\
Tumor grade & & \\
GI & $9.1 \%(2 / 22)$ & $59.1 \%(13 / 22)$ \\
G2 & $20.0 \%(I / 5)$ & $100.0 \%(5 / 5)$ \\
G3 & $12.5 \%(2 / 16)$ & $37.5 \%(6 / 16)$ \\
All samples & $11.6 \%(5 / 43)$ & $55.8 \%(24 / 43)$ \\
& $(95 \% \mathrm{Cl} \mathrm{5 \% -24 \% )}$ & $(95 \% \mathrm{Cl} 40 \%-71 \%)$ \\
\hline
\end{tabular}

Abbreviations: $\mathrm{Cl}$, confidence interval; $\mathrm{qPCR}$, quantitative polymerase chain reaction; smFGFR3, small-molecule FGFR3. it fails to produce similar results in urine, where the mutant to normal DNA ratio is much lower. The small-molecule FGFR3 assay overcomes this technical limitation by increasing analytical sensitivity, and detects as little as $0.02 \%$ mutant DNA. In the study presented here, while clinical sensitivity in urine for FGFR3 by quantitative PCR was $11.6 \%$, using the small-molecule FGFR 3 assay, we detected mutations in $55.8 \%$ of the same urine samples (Table 5), demonstrating that superior analytical sensitivity will ultimately improve clinical performance.

Importantly, clinical specificity in a hematuria population was not affected by the increased analytical sensitivity of the small-molecule $F G F R 3$ assay. Although the number of noncancer samples analyzed as part of this study was low, the results obtained with small-molecule $F G F R 3$ are consistent with quantitative PCR analysis of the same samples. In a separate study of approximately 700 patients with hematuria who were undergoing bladder cancer screening, we have shown that the clinical specificity and positive predictive value of FGFR 3 was $99.9 \%$ and $95.4 \%$, respectively, by quantitative PCR. ${ }^{17}$ Given the inherent specificity of DNA mutations for cancer and the results shown here, we would expect the smallmolecule FGFR3 assay to increase sensitivity significantly without losing specificity and retaining a very high positive predictive value. An assay with very high positive predictive value enables identification of patients with cancer but with very few false-positive results. In previous studies, we have also shown that the combination of FGFR 3 with other markers in one noninvasive assay also resulted in high sensitivity and a negative predictive value, a result that can be used to identify symptomatic patients that with high likelihood do not have cancer (high negative predictive value). ${ }^{8}$ The increased analytical sensitivity of the small-molecule FGFR3 assay would also be expected to increase sensitivity in this setting, increasing the negative predictive value and reducing false negative results.

The use of bodily fluids, such as urine, blood, or saliva, to detect cancer has potentially significant advantages over more invasive diagnostic methods for both the patient and the health care system. Currently, few assays based on bodily 
fluids have the clinical sensitivity required for diagnostic use. Other diagnostic urine assays for bladder cancer are being developed, some of which evaluate the levels or presence of metabolites or proteins in the urine or chromosomal changes in exfoliated cells. ${ }^{18-20}$ DNA-based assays have the advantage of being highly specific. The single molecule FGFR3 assay is unique in that it overcomes the limitation of detecting rare events in body fluids and provides physicians with a means to diagnose bladder cancer with high confidence.

\section{Acknowledgments}

The authors would like to thank Lei Li, Maria Campo, Lydia Anderson, and Christopher Cummings for technical support and Elizabeth Goodwin for editorial assistance. The authors would also like to thank Marsha A Moses and Robert S Langer for critical review of the manuscript.

\section{Disclosure}

JMM, SL, CAF, and APS are employees of Predictive Biosciences.

\section{References}

1. Siegel R, Ward E, Brawley O, Jemal A. Cancer statistics, 2011: the impact of eliminating socioeconomic and racial disparities on premature cancer deaths. CA Cancer J Clin. 2011;61(4):212-236.

2. Billerey C, Chopin D, Aubriot-Lorton MH, et al. Frequent FGFR3 mutations in papillary non-invasive bladder (pTa) tumors. Am J Pathol. 2001;158(6):1955-1959.

3. Cappellen D, De Oliveira C, Ricol D, et al. Frequent activating mutations of FGFR3 in human bladder and cervix carcinomas. Nat Genet. 1999;23(1):18-20.

4. van Rhijn BW, Lurkin I, Radvanyi F, Kirkels WJ, van der Kwast TH, Zwarthoff EC. The fibroblast growth factor receptor 3 (FGFR3) mutation is a strong indicator of superficial bladder cancer with low recurrence rate. Cancer Res. 2001;61(4):1265-1268.

5. van Rhijn BW, Vis AN, van der Kwast TH, et al. Molecular grading of urothelial cell carcinoma with fibroblast growth factor receptor 3 and MIB-1 is superior to pathologic grade for the prediction of clinical outcome. J Clin Oncol. 2003;21(10):1912-1921.

6. van Oers JM, Lurkin I, van Exsel AJ, et al. A simple and fast method for the simultaneous detection of nine fibroblast growth factor receptor 3 mutations in bladder cancer and voided urine. Clin Cancer Res. 2005;11(21):7743-7748.
7. Forbes SA, Bindal N, Bamford S, et al. COSMIC: mining complete cancer genomes in the Catalogue of Somatic Mutations in Cancer. Nucleic Acids Res. 2011;39 Database issue:D945-D950.

8. Fernandez CA, Millholland JM, Zwarthoff EC, Feldman AS, Karnes RJ, Shuber AP. A noninvasive multi-analyte diagnostic assay: combining protein and DNA markers to stratify bladder cancer patients. Research and Reports in Urology. 2012;4:17-26.

9. Zuiverloon TC, Tjin SS, Busstra M, Bangma CH, Boeve ER, Zwarthoff EC. Optimization of nonmuscle invasive bladder cancer recurrence detection using a urine based FGFR3 mutation assay. $J$ Urol. 2011;186(2):707-712.

10. Hernandez S, Lopez-Knowles E, Lloreta J, et al. Prospective study of FGFR3 mutations as a prognostic factor in nonmuscle invasive urothelial bladder carcinomas. J Clin Oncol. 2006;24(22):3664-3671.

11. Junker K, van Oers JM, ZwarthoffEC, Kania I, Schubert J, Hartmann A. Fibroblast growth factor receptor 3 mutations in bladder tumors correlate with low frequency of chromosome alterations. Neoplasia. 2008;10(1):1-7.

12. van Oers JM, Wild PJ, Burger M, et al. FGFR3 mutations and a normal CK20 staining pattern define low-grade noninvasive urothelial bladder tumours. Eur Urol. 2007;52(3):760-768.

13. van Rhijn BW, Lurkin I, Chopin DK, et al. Combined microsatellite and FGFR3 mutation analysis enables a highly sensitive detection of urothelial cell carcinoma in voided urine. Clin Cancer Res. 2003;9(1):257-263.

14. Diehl F, Schmidt K, Durkee KH, et al. Analysis of mutations in DNA isolated from plasma and stool of colorectal cancer patients. Gastroenterology. 2008;135(2):489-498.

15. Miyake M, Sugano K, Kawashima K, et al. Sensitive detection of FGFR3 mutations in bladder cancer and urine sediments by peptide nucleic acid-mediated real-time PCR clamping. Biochem Biophys Res Commun. 2007;362(4):865-871.

16. Junker K, Wolf M, Schubert J. Molecular clonal analysis of recurrent bladder cancer. Oncol Rep. 2005;14(2):319-323.

17. Karnes RJ, Fernandez CA, Shuber AP. A non-invasive multianalyte urine based diagnostic assay for urothelial cancer of the bladder in the evaluation of hematuria. Mayo Clinic Proceedings. In Press.

18. Issaq HJ, Waybright TJ, Veenstra TD. Cancer biomarker discovery: opportunities and pitfalls in analytical methods. Electrophoresis. 2011;32(9):967-975.

19. Lotan Y, Roehrborn CG. Sensitivity and specificity of commonly available bladder tumor markers versus cytology: results of a comprehensive literature review and meta-analyses. Urology. 2003;61(1):109-118.

20. Tilki D, Burger M, Dalbagni G, et al. Urine markers for detection and surveillance of non-muscle-invasive bladder cancer. Eur Urol. 2011;60(3):484-492. 


\section{Supplementary tables}

Table SI Cutoff values for each mutation site ${ }^{a}$

\begin{tabular}{|c|c|c|c|c|c|c|c|c|c|}
\hline \multirow[t]{2}{*}{ Mutation } & \multicolumn{2}{|l|}{ Exon 7} & \multicolumn{3}{|l|}{ Exon 10} & \multicolumn{4}{|l|}{ Exon 15} \\
\hline & R248C & S249C & G372C & S373C & Y375C & K652E & K652M & K652Q & K652T \\
\hline Projected cutoff, \% & 0.25 & 0.02 & 0.05 & 0.15 & 0.40 & 0.45 & 0.2 & 0.05 & 0.05 \\
\hline $\begin{array}{l}\text { Observed } \\
\text { background, \% }\end{array}$ & $0-0.15$ & 0 & $0-0.01$ & $0-0.08$ & $0.08-0.22$ & $0.06-0.37$ & $0-0.07$ & 0 & 0 \\
\hline
\end{tabular}

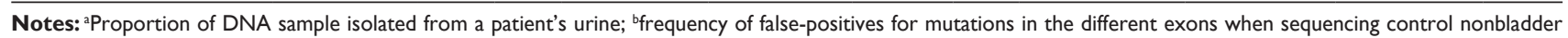
cancer human genomic DNA.

Table S2 Representative results of multiplex analysis of four patients with bladder cancer

\begin{tabular}{|c|c|c|c|c|c|}
\hline Patient & Reads/sample & Exon & Reads/exon & Mutant reads & $\%$ mutant \\
\hline \multirow[t]{3}{*}{ I } & $3284 I$ & 7 & 21730 & 22 & $0.10 \%$ \\
\hline & & 10 & 6979 & - & \\
\hline & & 15 & 3463 & - & \\
\hline \multirow[t]{3}{*}{2} & 29405 & 7 & $|808|$ & 7 & $0.04 \%$ \\
\hline & & 10 & 5193 & - & \\
\hline & & 15 & 3558 & - & \\
\hline \multirow[t]{3}{*}{3} & 21636 & 7 & 13915 & - & \\
\hline & & 10 & 4607 & - & \\
\hline & & 15 & 2922 & - & \\
\hline \multirow[t]{3}{*}{4} & 28556 & 7 & 19974 & - & \\
\hline & & 10 & 5337 & 23 & $0.43 \%$ \\
\hline & & 15 & 3075 & - & \\
\hline
\end{tabular}

Note: For this experiment, pooled DNA from urine samples of four individual patients was assayed for mutations in exons 7,10 , or 15.

\section{Publish your work in this journal}

Research and Reports in Urology is an international, peer-reviewed, open access journal publishing original research, reports, editorials, reviews and commentaries on all aspects of adult and pediatric urology in the clinic and laboratory including the following topics: Pathology, pathophysiology of urological disease; Investigation and treatment of urological disease; Pharmacology of drugs used for the treatment of urological disease. The manuscript management system is completely online and includes a very quick and fair peer-review system, which is all easy to use. Visit http://www.dovepress.com/testimonials.php to read real quotes from published authors 